\title{
Insertion force of micro-probe into brain phantom
}

\author{
Lihong $\mathrm{Li}^{1,2, \mathrm{a}}$, Jun Zhou ${ }^{1,2, \mathrm{~b},{ }^{*},}$, Yan $\mathrm{Li}^{1,2, \mathrm{c}}$, Fuqian Chen ${ }^{1,2, \mathrm{~d}}$, Yongbo Wang ${ }^{1,2, \mathrm{e}}$ \\ ${ }^{1}$ School of Mechanical Engineering, Shandong University, Jinan 250061, China; \\ ${ }^{2}$ Key Laboratory of High Efficiency and Clean Mechanical Manufacture (Shandong University), \\ Ministry of Education, Jinan 250061, China \\ axiaoxi900727@163.com, 'bhoujun@sdu.edu.cn, ${ }^{c} 375998051 @ q q . c o m$,

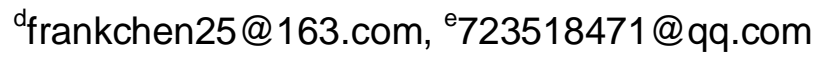

*Corresponding author: Jun Zhou, E-mail: zhoujun@sdu.edu.cn, Tel.: +86-531-88392047.

Keywords: Insertion force, Micro-probe, Brain phantom, Straight and reciprocating implanted Abstract. The research of forces during probe insertion into soft tissue is extremely important for accuracy of electrode implantation in deep brain stimulation. In this paper, to further understand the mechanics of probe-tissue interaction, straight implanted experiments and reciprocating implanted experiments were conducted in an artificial brain tissue substitute. The results illustrate that in the straight implanted experiments the insertion force of probe is accumulating while the insertion depth is increasing and the peak force increases with the insertion velocity range of $0.5 \sim 3.0 \mathrm{~mm} / \mathrm{s}$ in our work. Moreover, reciprocating implanted can effectively release the stress on the probe which helps to improve the direction of the probe so as to improve target localization accuracy.

\section{Introduction}

During the past 20 years deep brain stimulation has become a routine method for the treatment of advanced Parkinson's disease (PD), leading to striking improvements in motor function and life quality of PD patients. The success of the therapy largely depends on the precise implantation of the stimulation electrode, which is also the key factor of reducing complications and the side effects [1]. Precise implantation of the stimulation electrode depends on a thorough understanding of the mechanics of needle-tissue interaction, therefore, the interaction between needle (or other similar surgical tools) and soft tissue has been a subject of significant research effort in recent years [2-4]. Witter et al. [5] conducted experiments of needle insertion into a swine brain, focused on the insertion phase preceding puncture of the brain meninges and obtained a very accurate prediction of the needle force. Welkenhuysen et al. [6] investigated the effect of insertion velocity on long term tissue response and insertion mechanics. In their study a dummy silicon parylene-coated probe was used and implanted in the rat brain at $10 \mu \mathrm{m} / \mathrm{s}$ or $100 \mu \mathrm{m} / \mathrm{s}$ to a depth of $9 \mathrm{~mm}$. Casanova et al.[7] provided a new methodology to evaluate the needle force and friction stress during needle insertion and retraction at varying insertion velocity in a tissue phantom material and in rat brain and drew a conclusion that force derived friction stress decreased with increasing insertion velocity.

In those above studies, however, the insertion and implantation of devices like needles, probes, and electrodes whether into biological tissue or artificial tissue were straight implanted, while reciprocating implanted was very little discussion. This article mainly focuses on force analysis of the micro-probe implanted into brain phantom in the manner of straight implanted and reciprocating implanted. The experimental results illustrate that in the straight implanted experiments the insertion force of probe is accumulating while the insertion depth is increasing and the peak force 
increases with the insertion velocity range of $0.5 \sim 3.0 \mathrm{~mm} / \mathrm{s}$ in our work. Moreover, reciprocating implanted can effectively release the stress on the probe which helps to improve the direction of the probe so as to improve target localization accuracy.

\section{Materials and Methods}

\section{Preparation of Tissue Phantom}

It is rather difficult to perform probe implantation experiments in vivo for practical as well as ethical reasons. Therefore, artificial biological substitute tissue has been widely used in clinical and experimental research for its practical advantages, such as high transparency, visuality, stability, availability. Gelatin gel is often used as substitute material for brain tissue e.g. in literature [8]. In this paper the insertions were done into gelatin phantom made from edible gelatin powder and mixed to a ratio of $1.3-\mathrm{ml}$ gelatin to $20-\mathrm{ml}$ boiling water. Furthermore, glycerin was added as a kind of additives to adjust the viscosity of gelatin gel. When the volume ratio of gelatin powder, glycerin, deionized water is 1.3:0.9:20, the hardness and viscoelasticity of the gel is most close to the swine brain tissue, which has already been verified in our previous study [9].

\section{Experimental Set-up}

The insertions were conducted using a custom built apparatus, depicted in Fig. 1. Insertion force data was collected using a NANO17 sensor system (ATI Industrial Automation, USA). In this sensor system, the data was filtered with an in-built 2nd order, $5.1 \mathrm{kHz}$ low-pass anti-aliasing filter, and acquired at $5 \mathrm{kHz}$ via a data acquisition card (NI PCI-6220, National Instruments). A linear actuator (M-L01.4A1, Physik Instrumente, Germany) with $2 \mu \mathrm{m}$ resolution and $25 \mathrm{~mm} / \mathrm{s}$ maximum speed was used to control and record the displacement. The experiment was documented by a microscope system (DH-HV3151UC-1 ML, Daheng IMAVISION, China).

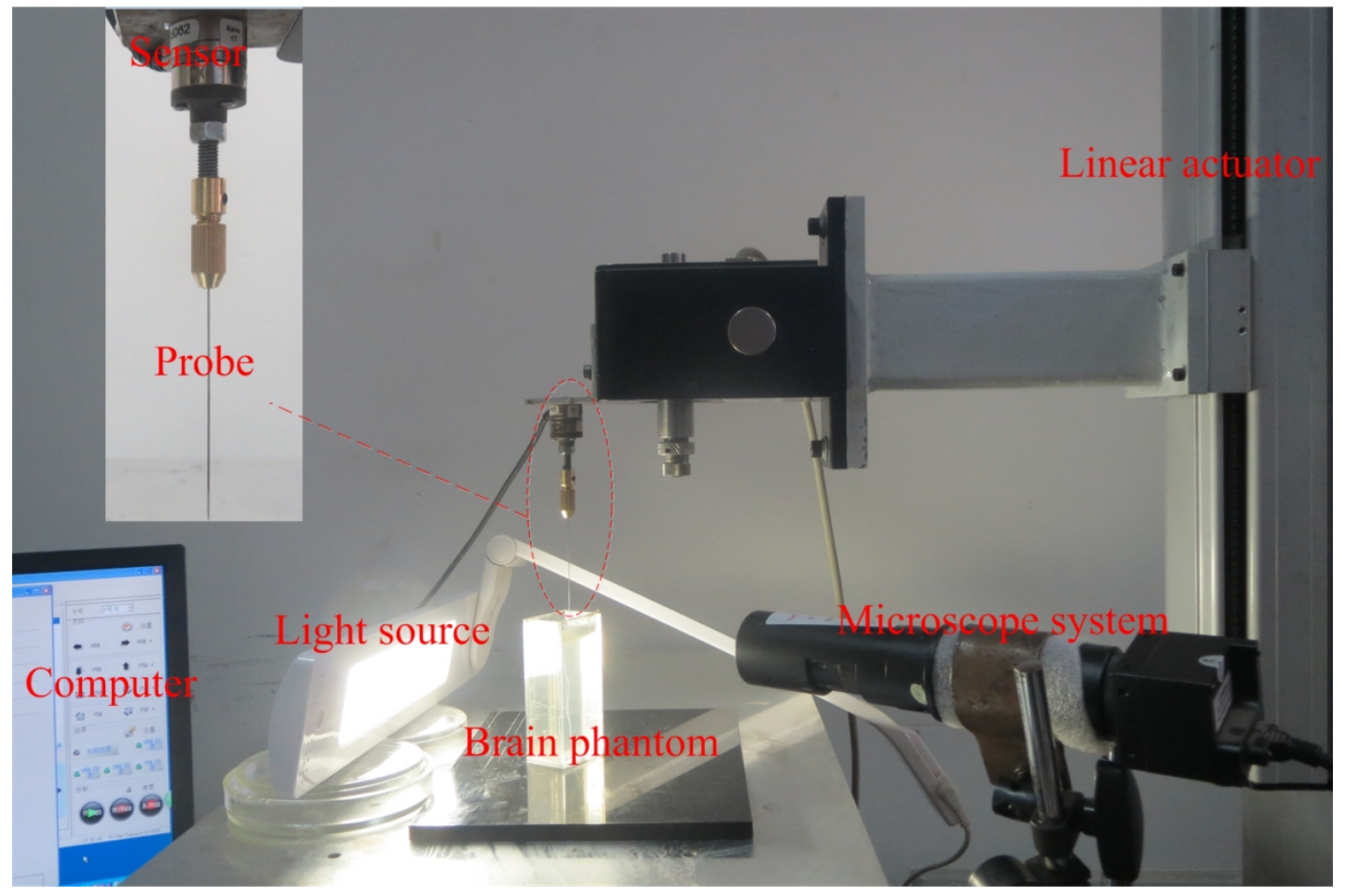

Fig. 1 Experimental set-up

\section{Experimental Procedure}

A $0.5 \mathrm{~mm}$ diameter, $81 \mathrm{~mm}$ long stainless steel probe with conical tip was inserted into the gelatin phantom. Before insertion, the probe was brought close to the surface of the phantom rapidly. Then, the fine-adjustment GUI of the insertion apparatus was applied to bring the probe in contact with 
the phantom surface with the help of the microscope.

During the straight implanted tests, the probe was actuated to reach a depth of $15 \mathrm{~mm}$ at a constant velocity for each test. Insertion forces were recorded for insertion velocities from $0.5 \sim 3 \mathrm{~mm} / \mathrm{s}$ (the interval is $0.5 \mathrm{~mm} / \mathrm{s}$ ). Experiments were repeated 3 times at the same area of different test points for each insertion velocity. All tests were performed at room temperature.

Reciprocating implanted means that when the probe is inserted into the middle position of the tissue, retract a certain distance and then perform straight movements until the tip arrives at the target location. The current reciprocating implanted tests were done in gelatin phantom with the same insertion and retraction velocity $(1 \mathrm{~mm} / \mathrm{s})$ and the same final insertion depth $(15 \mathrm{~mm})$, and the only variable was the retraction distance. The reciprocating implanted process was divided into three stages: (1) The probe was actuated to reach a depth of $12.5 \mathrm{~mm}$ at a constant velocity $(1 \mathrm{~mm} / \mathrm{s})$ and then stopped insertion. (2) The probe was retracted a certain distance $5 \mathrm{~mm}, 7.5 \mathrm{~mm}$ and $10 \mathrm{~mm}$ respectively with 10 s hold time. (3) Further insertion of the probe into phantom to the final location.

\section{Results}

\section{Mechanics Analysis of Straight Implanted Process}

Straight implanted tests of varying insertion velocities aimed at detailing analysis the variation of insertion force and the influence of insertion velocity on the forces. Insertion force is defined here as the force acting on the probe in the direction of insertion. As will be discussed in the following sections, the insertion force consists of stiffness force, cutting force and friction force.

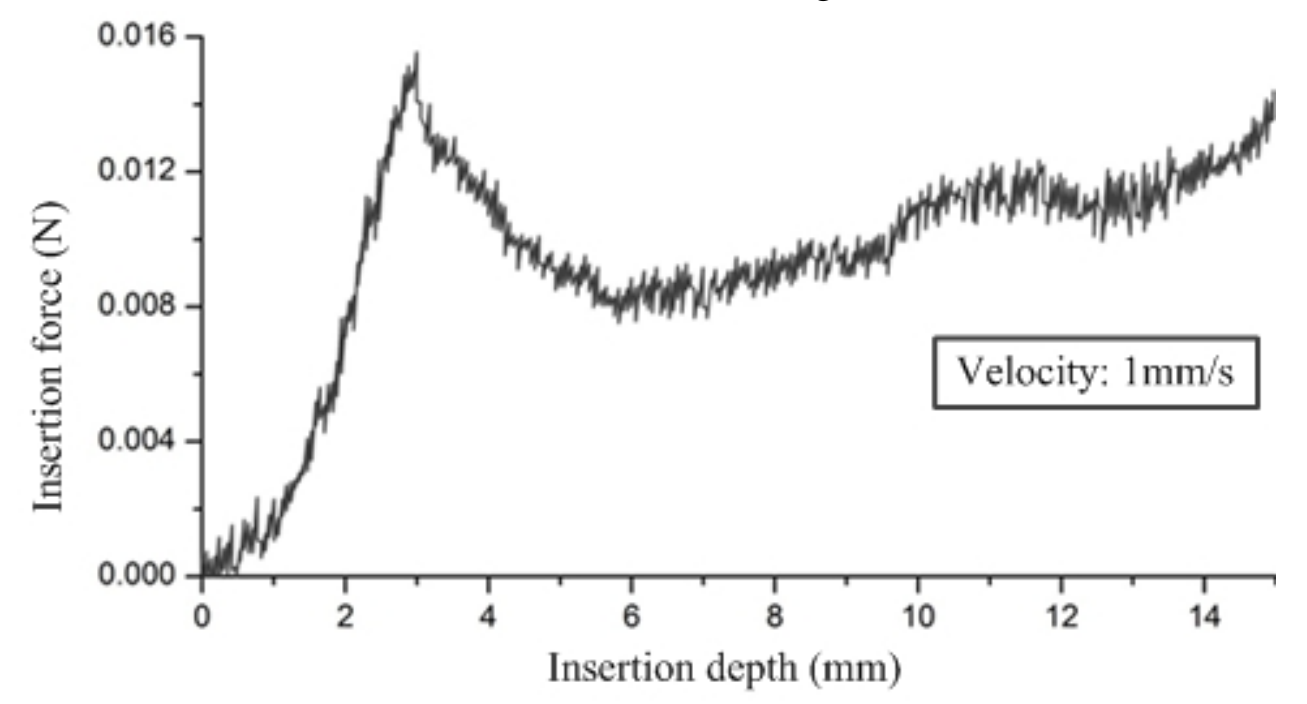

Fig. 2 Typical insertion force versus insertion depth recorded in this study

The general behavior of the insertion force versus insertion depth in this study is shown in Fig. 2, which is consistent with the one recorded by Wittek et al. [5] for needle insertion into swine brain. It can be seen from Fig. 2 after the probe touches the phantom surface, insertion force increases with the insertion depth approximately linear rise due to the elasticity of gelatin phantom. At this time the insertion force is shown as stiffness force. At the instant of gelatin surface puncture, the stiffness force reaches its maximum, which shows a peak force on the curve. After the probe tip pierces gelatin surface, insertion force drops sharply and changes to the total force of friction force and cutting force. The reasons for insertion force dropping sharply are the elasticity and rheological property of the gelatin. Then insertion force is gradually increasing with the insertion depth as a result of the existence of friction. At post-puncture stage insertion force is very complex. Probe tip and probe shaft contact with the tissue, and the tip cuts the tissue forming cutting force which generating tissue damage. Friction force exists on the probe shaft and tip contacting with the tissue. 
Friction force on the shaft doesn't destroy tissue and the friction force on the tip is smaller, therefore, insertion force within the tissue can be regarded as the cutting force and the probe shaft friction force.

Generally speaking, cutting force is a constant value once the tissue is given, having nothing to do with insertion depth [4]. It can be concluded that the friction force is proportional to insertion depth, which is consistent with the modified Karnopp friction model [3]. For the stiffness force, the peak force shows a tendency of increasing with the insertion velocity as summarized in Fig. 3. Due to elasticity and rheological property of the phantom tissue, the probe will be in an unstable status at the instant of puncture thus affecting the accuracy of the subsequent puncture path and increasing the offset of the tip. In the straight implanted process, therefore, reducing the insertion velocity appropriately can decrease the offset of puncture path caused by excessive stiffness force so as to improve target localization accuracy.

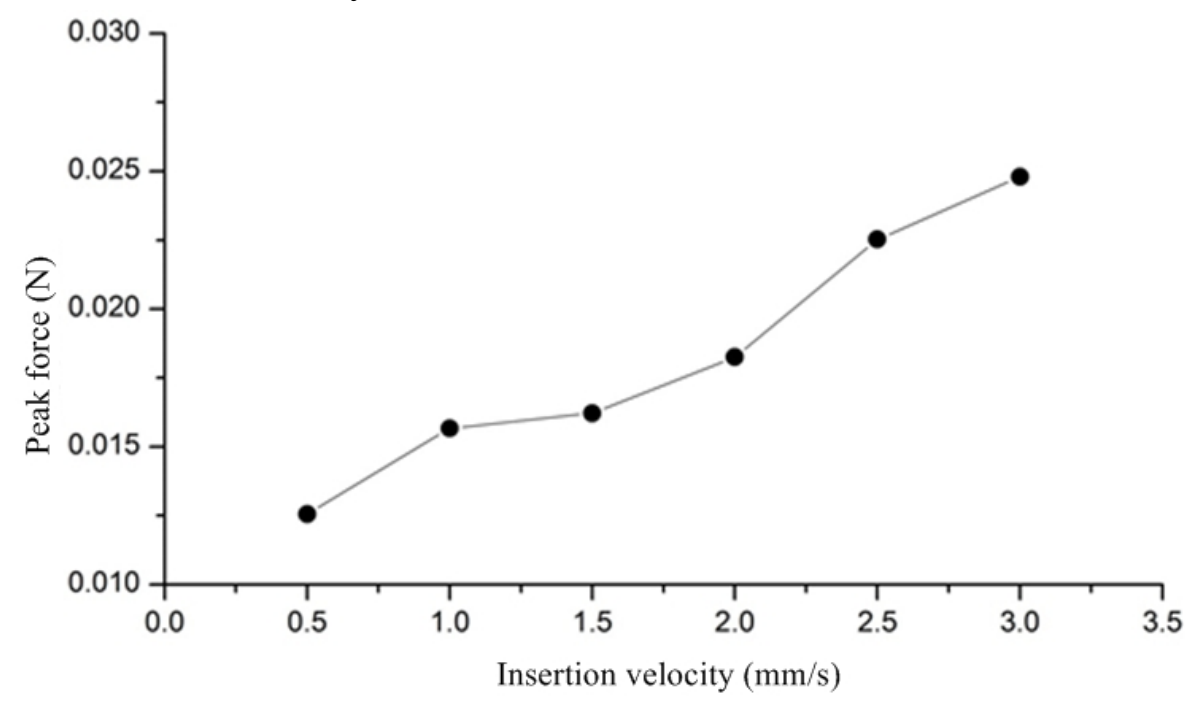

Fig. 3 The relationship between the peak force and insertion velocity

For the infinitesimal deformation of tissue, the characteristic is linear elastic, while the linear model was not applied to the stiffness force. Hence, in this paper we used the quadratic polynomial model and the nonlinear spring model to fit the stiffness force data in Matlab and made comparison and analysis for the two models.
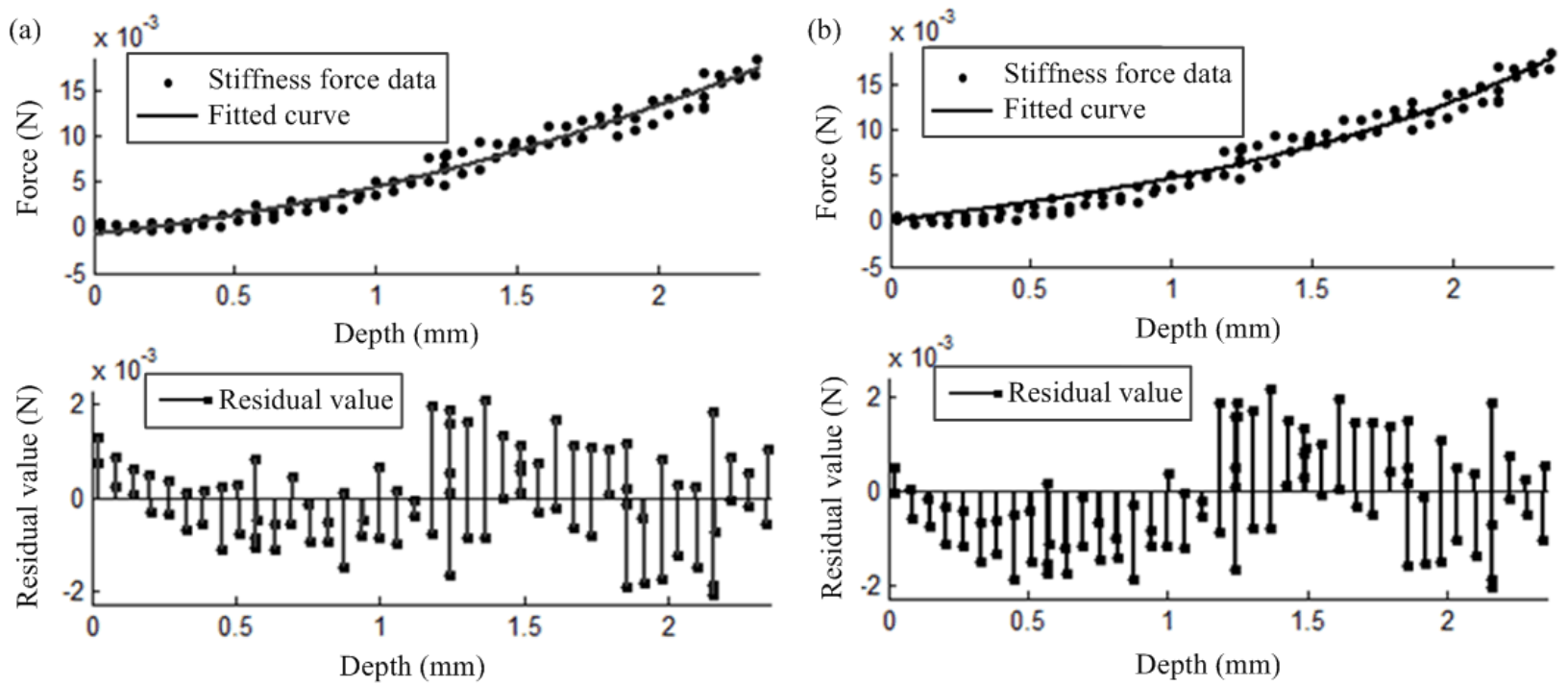

Fig. 4 The fitting curve and residual distribution curve of (a) quadratic polynomial model and (b) nonlinear spring model 
Fig.4(a) and Fig.4(b) show that the stiffness force data points are evenly distributed near the quadratic polynomial model fitting curve while for the nonlinear spring model residual values are negative and there is no randomness within the $0 \sim 1.2 \mathrm{~mm}$ depth. In the range of $1.2 \sim 2.3 \mathrm{~mm}$, the residual values of the two kinds of model fitting are almost the same and have no much difference. For the two kinks of model the larger residual values are concentrated during the second half of pre-puncture, which proves that before the piercing, the probe exists instability and the stiffness force presents relatively large fluctuation. The residual sum of squares (SSE) of quadratic polynomial model is an order of magnitude lower than that of nonlinear spring model and the R-square of quadratic polynomial model fitting curve is closer to 1 . These data are listed in Table 1 . Summing up the above analysis, a conclusion can be drawn that for small deformation quadratic polynomial model is more suitable for describing stiffness force.

Table 1 Fitting parameters and fitting degree of the models

\begin{tabular}{|c|c|c|c|c|}
\hline \multirow[t]{2}{*}{ Model } & \multicolumn{2}{|c|}{$\begin{array}{l}\text { Quadratic Polynomial Model } \\
\qquad f(x)=a_{0} x^{2}+a_{1} x\end{array}$} & \multicolumn{2}{|c|}{$\begin{array}{l}\text { Nonlinear Spring Model } \\
\qquad f(x)=\frac{x}{a x+b}\end{array}$} \\
\hline & $a_{0}$ & $a_{1}$ & $a$ & $b$ \\
\hline Fitted value & $0.00234 \mathrm{~N} / \mathrm{mm}^{2}$ & $0.00198 \mathrm{~N} / \mathrm{mm}$ & $-62.0 \mathrm{~N}$ & $277 \mathrm{~mm} / \mathrm{N}$ \\
\hline SSE & \multicolumn{2}{|c|}{$9.32 \mathrm{e}-5$} & \multicolumn{2}{|c|}{$1.11 \mathrm{e}-4$} \\
\hline $\mathrm{R}$-square & \multicolumn{2}{|c|}{0.966} & \multicolumn{2}{|c|}{0.959} \\
\hline
\end{tabular}

\section{Mechanics Analysis of Reciprocating Implanted Process}

The reciprocating implanted process was divided into three stages as described in Section 2.3. Since the insertion force during the first phase was same with the force in straight implanted process, in this part we only analyzed the data obtained from the second and third phase. A typical curve of insertion force versus time obtained from 5-mm retraction distance is described in Fig. 5.

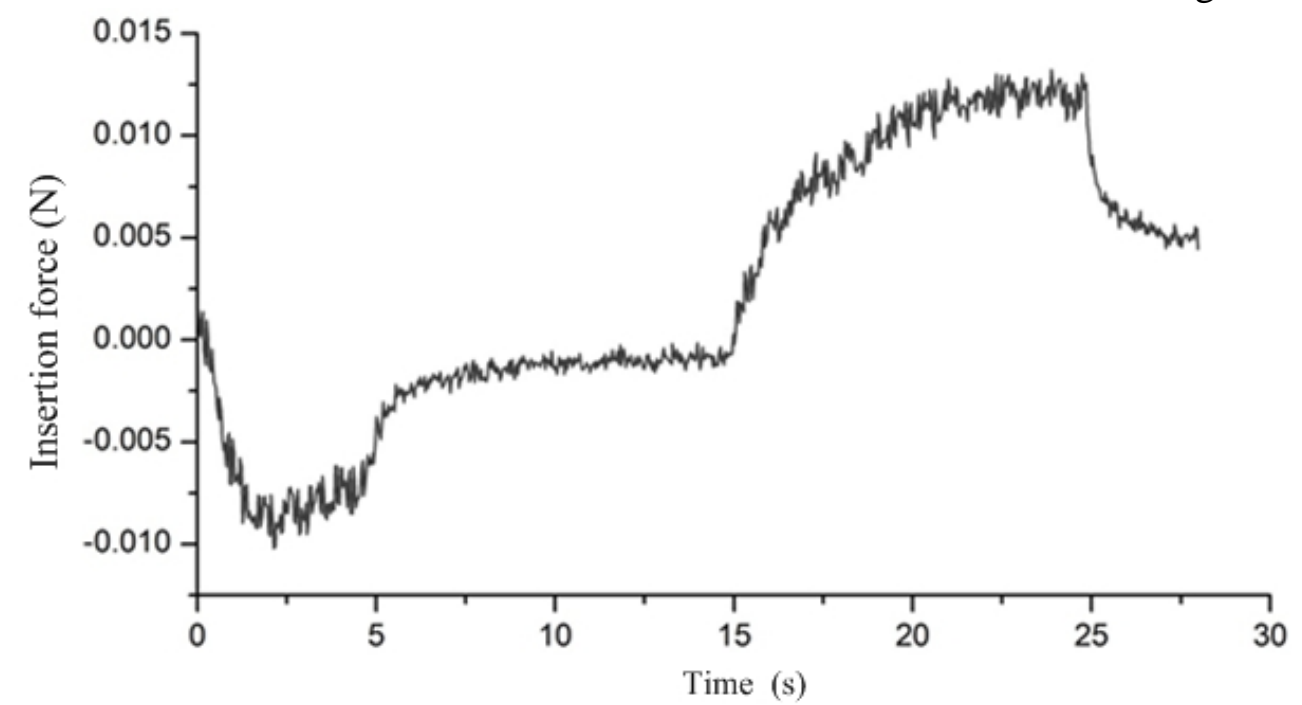

Fig. 5 A typical curve of insertion force versus time when the retraction distance is $5 \mathrm{~mm}$

As can be seen in Fig. 5 the force value of initial point of retraction phase is about $0.002 \mathrm{~N}$, which is a constant value caused by tissue relaxation after stopping insertion in first phase. There only existed coulomb friction during probe retraction phase, which having no effect on tissue damage. At the beginning due to viscous and damping of the tissue, the tissue and the probe did not produce relative motion, at this time the friction force was static friction force. Then, when probe and tissue produced relative motion, the friction force would translate into dynamic friction force. Dynamic friction would decrease with the increasing velocity in low-speed stage. The friction model based 
on the experimental data is shown in Fig. 6 . The curve of $5 \mathrm{~s} \sim 15 \mathrm{~s}$ is the stress relaxation stage of 10 seconds and after $15 \mathrm{~s}$ it stands for repeating insertion phase. When the probe inserted to the final location (15 mm depth), the maximal force was $0.0129 \mathrm{~N}$ decreasing about $20 \%$ than the straight implanted under the same insertion velocity $(1 \mathrm{~mm} / \mathrm{s})$. Thus, we can draw a conclusion reciprocating implanted can reduce the insertion force within the tissue, resulting in a certain effect on improving the target localization accuracy.

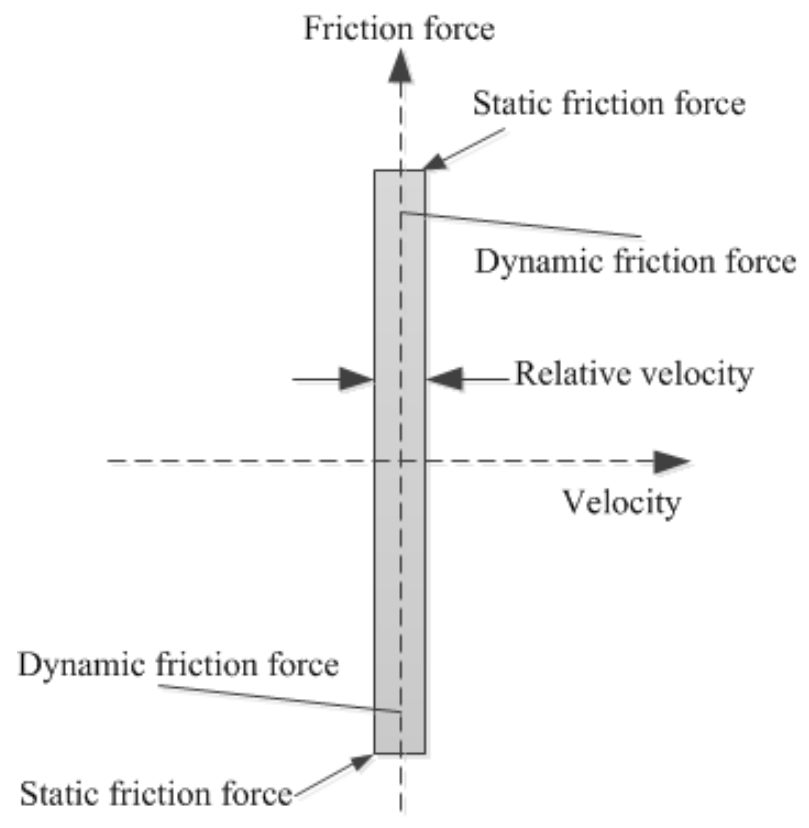

Fig. 6 Friction force model based on experimental data

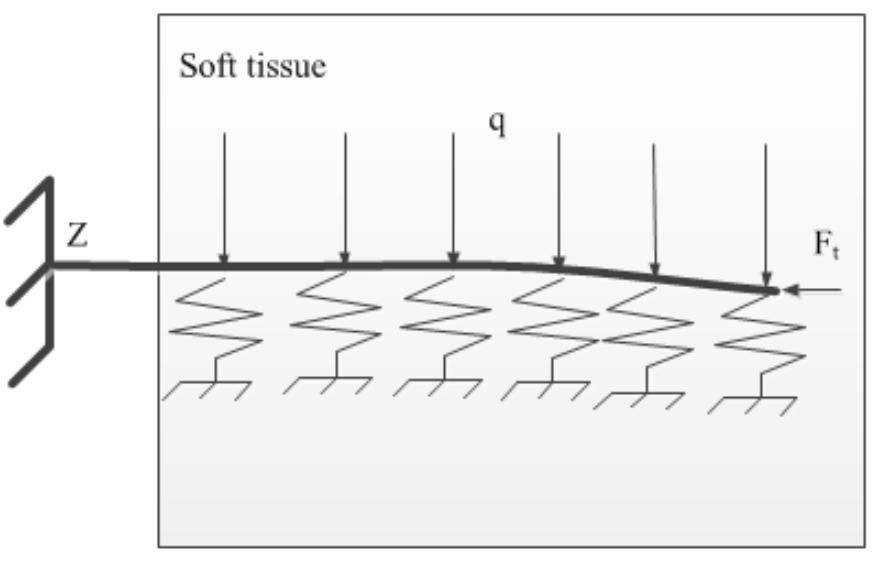

Fig. 7 The cantilever beam model

When the probe is inserted into tissue, the pressure difference (on both sides of probe shaft and tip) will cause offset on the puncture path, so that the target positioning error is gradually enlarged. Through analyzing the probe based on the cantilever beam model, we can simplify the micro-probe as a base fixed cantilever beam as shown in Fig. 7. We can see that the probe will occur a certain bending due to the influence of axial load and pinpoint load distribution and the deformation of $\mathrm{Z}$ direction can be neglected due to the high stiffness. The offset of probe tip increases constantly since we can't guarantee the probe is strictly perpendicular to the base. Nevertheless, reciprocating implanted can make tissue relaxation during probe retraction phase and release the stress on the probe at the same time, resulting in efficiently reducing the offset with respect to the ideal puncture path. And the ultimate target error could also be able to get a certain control, so the reciprocating implanted is a good target control strategy.

\section{Conclusions}

In this study, we performed straight implanted experiments and reciprocating implanted experiments in gelatin phantom in order to further understand the mechanics of probe-tissue interaction. Straight implanted experiments with insertion velocities from $0.5 \mathrm{~mm} / \mathrm{s}$ to $3 \mathrm{~mm} / \mathrm{s}$ and $15 \mathrm{~mm}$ insertion depth were carried out and the tendency of the insertion force was analyzed in detail. It was found that the stiffness force peak decreased with the insertion velocity decreasing, thus reducing the unnecessary shaking at the instant of puncture. We fitted the stiffness force experimental data using the quadratic polynomial model and nonlinear spring model in Matlab software and draw a conclusion that in our work the former was superior to the latter when the tissue was small deformation. Meanwhile reciprocating implanted experiments were also conducted. It was found that at the just beginning of probe retraction stage there was no relative motion 
between the tissue and the probe and the static friction force increased sharply. While there was relative motion, the friction force would translate into dynamic friction force and the dynamic friction force presented a tendency of decreasing as velocity increasing. Experimental results show that the force curve of reciprocating implanted is smoother than the straight implanted, and the former can effectively reduce the unstable state of probe and improve the target localization accuracy.

\section{Acknowledgments}

This research was financially supported by the National Natural Science Foundation of China (Grant No. 51375268) and the Independent Innovation Foundation of Shandong University (Grant No. 2012ZD009).

\section{References}

[1] S. Breit, J.B. Schulz, and A.L. Benabid: Deep brain stimulation, Cell Tissue Res. Vol.318(2004), p.275-288

[2] S.P. DiMaio, S.E. Salcudean: Interactive simulation of needle insertion models, IEEE Trans. Biomed. Eng. Vol.52(2005), p.1167-1179

[3] A.M. Okamura, C. Simone, and M.D. O'Leary: Force modeling for needle insertion into soft tissue, IEEE Trans. Biomed. Eng. Vol. 51(2004), p.1707-1716

[4] D.J. van Gerwen, J. Dankelman, J.J. van den Dobbelsteen: Needle-tissue interaction forces-A survey of experimental data, Med. Eng. Phys. Vol.34(2012), p. 665-680

[5] A. Wittek, T. Dutta-Roy, Z. Taylor,A. Horton, T. Washio, K.Chinzei, K. Miller: Subject-specific non-linear biomechanical model of needle insertion into brain, Comput. Meth. Biomech. Biomed. Eng. Vol.11(2008), p.135-146

[6] M. Welkenhuysen, A. Andrei, L. Ameye, W. Eberle and B. Nuttin: Effect of insertion speed on tissue response and insertion mechanics of a chronically implanted silicon-based neural probe, IEEE Trans. Biomed. Eng. Vol.58(2001), p. 3250-3259

[7] F. Casanova, P.R. Carney, M. Sarntinoranont: In vivo evaluation of needle force and friction stress during insertion at varying insertion speed into the brain, J. Neurosci. Meth. Vol.237(2014), p.79-89

[8] J.A. Engh, G. Podnar, S.Y. Khoo, and C.N. Riviere: Flexible needle steering system for percutaneous access to deep zones of the brain, Proc. 32nd IEEE Northeast Bioeng. Conf. (2006), p.103-104

[9] P.C. Liu: Research of the implantation drive strategy of deep brain simulation by intracranial microelectrodes, Master's thesis, Shangdong University (2014) 